\title{
The development of a virtual laboratory based on Unreal Engine 4
}

\author{
D A Sheverev ${ }^{1}$ and I N Kozlova ${ }^{1}$ \\ ${ }^{1}$ Samara National Research University, Moskovskoye shosse 34A, Samara, Russia, 443086
}

\begin{abstract}
In our days we have the problem in the system of training highly skilled engineering staff- the impossibility of teaching students high-tech equipment because of its fragility and high cost. The solution of this problem is the creation of a virtual laboratory works, which represent a computer simulation of the real hardware - the simulator. The main advantages of virtual laboratories is the lack of significant costs for the buy, repair and maintenance of expensive equipment, increased visibility of the processes of the explored process, unlimited up building the virtual laboratory's functional and etc. At the moment the laboratory work is developed and its purpose is to research the hysteresis of linear deformations of piezoelectric elements and it is tested in real educational process. Further development of this work is to increase the number of training models, and of course as support for mobile devices.
\end{abstract}

\section{Introduction}

The main task of education was and always is the development of the student's personality in conjunction with the mastery of knowledge, skills and abilities that can be applied in practice [1-5]. In the system of higher professional education, especially for the training of engineering staff, it is extremely important to obtain practical skills in working with equipment [6,7]. And the main opportunity is to receive them in the process of training is the performance of laboratory work.

We have the first problem of the existing system of education. For training highly qualified staff for advanced areas of the economy, it is necessary to develop skills in high-tech equipment, for example, equipment is used in the production of integrated circuits. In view of the extremely high cost of such equipment and consumables to it, as well as the complexity of operation (requirement of special skills for its use, the possibility of failure due to incorrect use), doing laboratory work on a regular basis is extremely difficult.

Also, in our days, the activity of higher educational institutions is in priority of the organization of the scientific research, while the provision of the educational process by the laboratory fund is financed in a limited way. Timely updating of the laboratory base is a costly and extremely inertial measure, the effectiveness of which is not obvious. However, employers as one of the criteria put forward exactly the skills of working on modern equipment.

The solution of this problem may be the simulation of laboratory equipment. Simulation which is built on well-known mathematical models of various physical and chemical phenomena can be approximated to the actual experiment. And such approach will make it possible, without significant expenditures and significantly expand the range of practical issues to improve the knowledge of material on the topic of the discipline. 
Another important aspect is the fact that virtual laboratory's works can help to solve another problem. We are talking about a rapid increase in the abstractness of the processes under investigation, which further increases the gap between theory and practice.

The use of virtual laboratories can help to relieve tension in this contradiction "theory-practice", it allows forming primary (basic) skills of working with research high-tech equipment without wear and tear of the latter, establishes and / or maintains the connection of theoretical knowledge with practical activity develops and consolidates interest to the chosen professional sphere.

The student's interest in professional activities, in turn, allows:

- to reveal the potential of students, because its promotes the development of the ability to set, formalize and solve problems, generate new ideas and create new knowledge;

- to improve the efficiency of the research work of the unit, because an enthusiastic student wants to expand the range of tasks from standard training to unique research.

- to show students a culture of production, because it opens the prospects for a systematic approach to solving the tasks.

\section{What is a virtual laboratory and what is needed to create it?}

The virtual laboratory is a simulation of working with the real equipment based on already known experience and mathematical models. It includes three-dimensional models of devices and appliances, environments (walls, doors, tables, chairs, etc.), which are recreated with high accuracy and realism. Also to enhance the realism all models are provided with detailed textures. Examples of such models you can see in Figure 1.
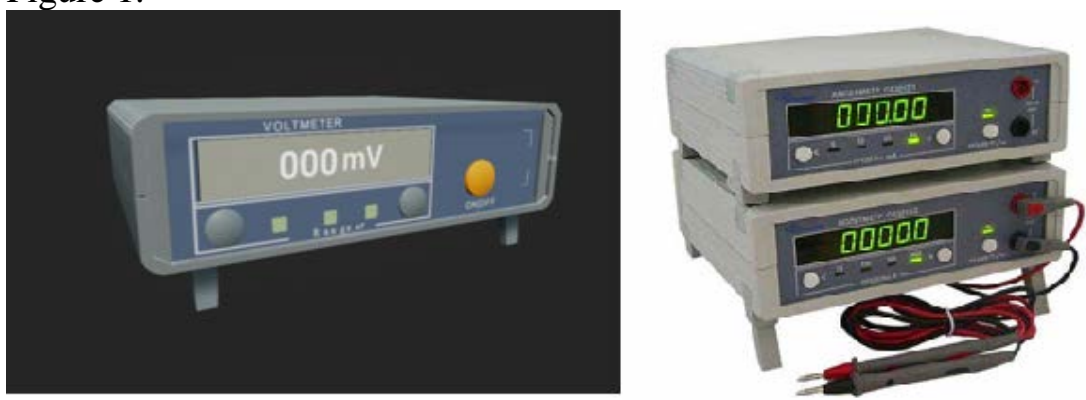

Figure 1. Voltmeter model in the left and real reference on right.

In the virtual laboratory, we use the first person's view of the abstract character to control the situation in the virtual laboratory, using the keyboard and mouse, similar to computer games.

In order to "revive" the virtual laboratory is necessary to write the interaction logic of user with it, as well as the objects of the laboratory with each other. For example, when approaching the door, it should open, etc.

Also in the virtual laboratory included the visualization of various effects which are studied in the work. For example, in Figure 2 we can see an example of a crystal lattice inside, by means of which we can see the crystal structures.

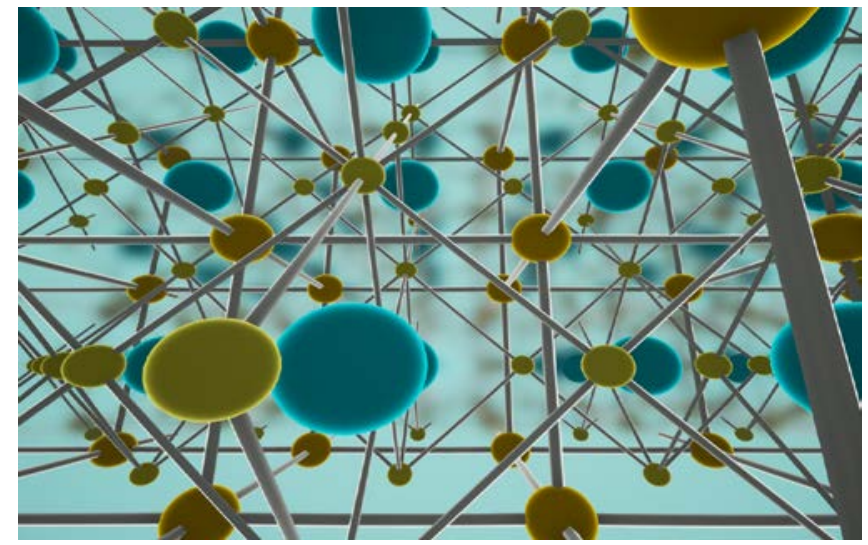

Figure 2. Crystal lattice visualization. 
The important part is the methodological support is presented in the form of an interactive methodology that can be called at any time. The example of methodological guidance is shown in Figure 3.

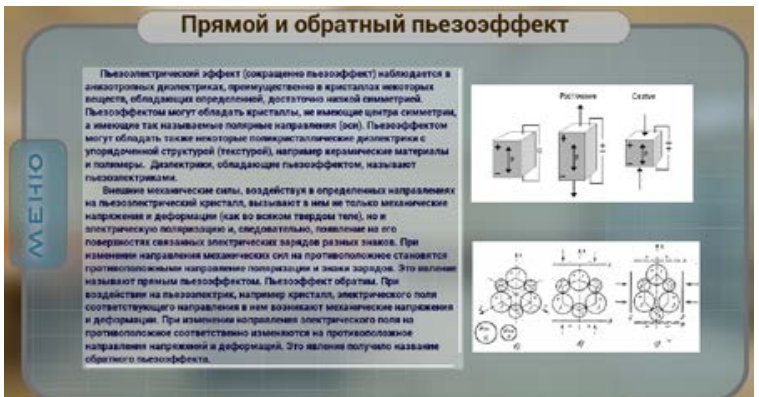

Figure 3. Methodical instructions in the virtual laboratory.

\section{Advantages of virtual laboratories}

What is the advantage of virtual laboratory work in compare with the real ones? I just want to say that their use does not exclude the work with real equipment, but is in addition to it.

For the training of highly qualified staff for the advanced fields of the economy (micro and nanotechnology), it is necessary to develop skills in high-tech equipment (such as plasma-chemical etching facilities, electronic lithography, precise analytical equipment, etc.). In view of the extremely high cost of such equipment and consumables to it, and the complexity of operation (requiring special skills for its use, the possibility of failure due to incorrect use), laboratory work on a regular basis is extremely difficult. Virtual laboratory work can solve the problem. Virtual equipment does not break down, does not wear out and does not require materials for the work.

Another important advantage is an increase in the visibility of processes and phenomena. Progress now dictates the high speed of development of technologies in the direction of their complication and increase of abstractness of the studied processes. High abstractness can reduce the effectiveness of understanding the material in the preparation of highly skilled engineering staff. The use of virtual reality solves this problem, allowing visualizing the physical and chemical processes and visualizing them. The motivation of students to the learning process is increased, making it interesting, and also adding game moments.

Also, the advantages of virtual laboratory work is unlimited increase in functionality, the addition of new laboratory works and other methodological developments that require visualization without changing the hardware complex or with irrelevant additions.

\section{Why Unreal Engine 4?}

For realizing the software component of the project is used the game engine "Unreal Engine 4" from Epic Games. It is used for arranging objects at the level, writing logical and mathematical operations, adjusting lighting and materials (textures), and final assembly of the application. Advantages of its using are:

- providing an accurate lighting model makes it possible to achieve a realistic picture;

- visual programming system "Blueprint" accelerating the development process and eliminating necessity for knowledge of programming languages. We can see it in Figure 4;

- extensive set of the development tools.

\section{What is finished?}

At the moment, the development of the laboratory work "Investigation of the hysteresis of linear deformations of piezoceramic elements" in the discipline "Physical and chemical bases of micro- and nanotechnology" is being actively carried out. The basic principles of developing logical and mathematical algorithms, applications based on them and 3D visualization are mastered. By now, the performance of laboratory work is carried out using a keyboard and mouse and it is the management of an abstract character in the first person. 


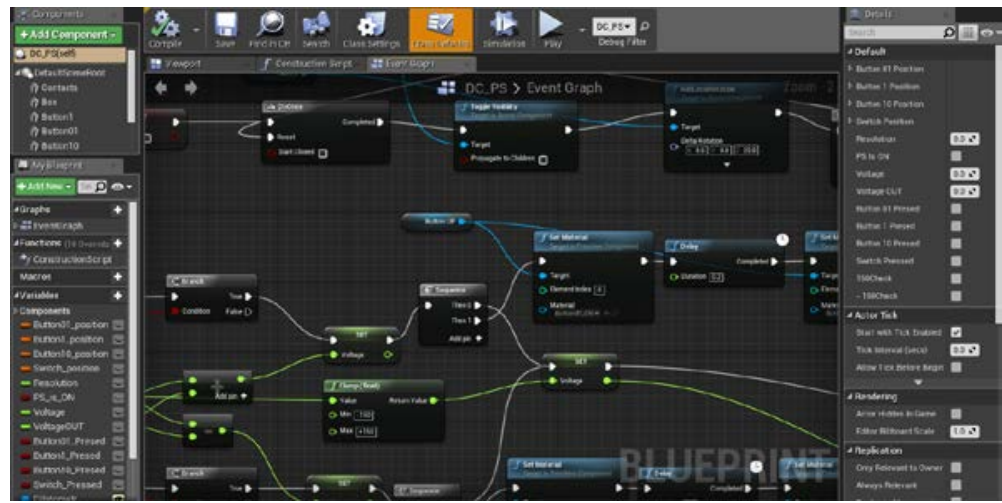

Figure 4. Visual programming system "Blueprint".

The room in which the laboratory work is divided into 3 sections:

1. The training rooms. At the beginning of the virtual laboratory work is necessary undergo training in the basics of control and interaction with objects;

2. Room of demonstrations. It contains extensive displays showing the monitoring process (and in particular the hysteresis of piezoceramics) in different levels, starting from a general view of the piezoelectric element, ending with the crystal lattice;

3. Room with laboratory equipment. It contains laboratory bench and rack with the samples is shown in Figure 5.

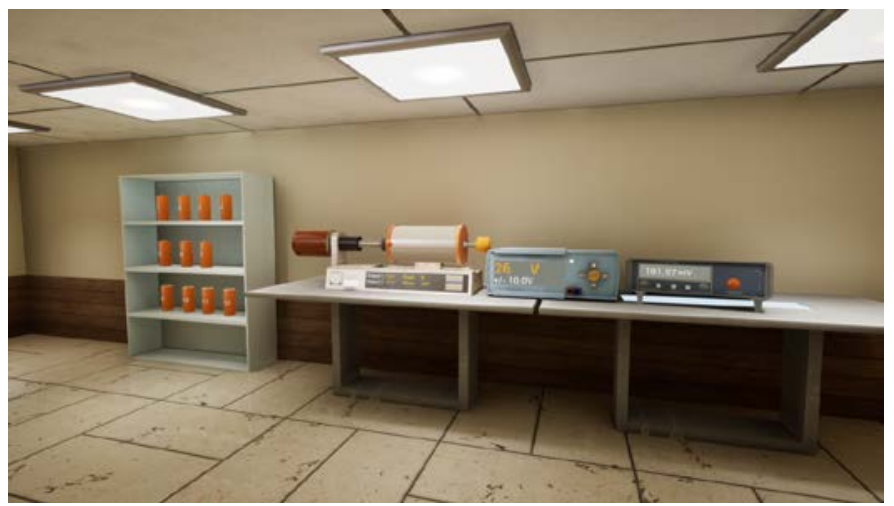

Figure 5. Laboratory bench and rack with the samples.

In any time the student can access the electronic methodical instructions and to study the theory or to ask a drone - assistant is presented in Figure 6.

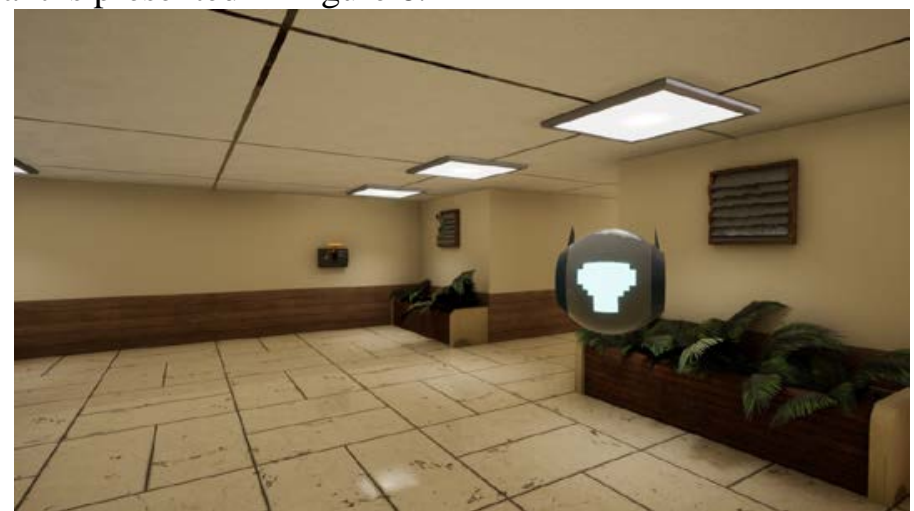

Figure 6. Drone - assistant.

The application is at the testing stage in the conditions of a real educational process. Also work is being done on the sound accompaniment of laboratory work, which is an important factor that allows using an additional channel of human perception. 


\section{What is next?}

The next stage in the development of this work is the introduction of virtual reality technologies, which will bring the level of perception of the material to a fundamentally new level. When using the virtual reality helmet and special controllers, the user creates a "full immersion" effect, which makes it easier to relate virtual equipment to the real one.

The virtual laboratory, created as a result of the completion of this project will consist of two parts: hardware and software.

The hardware consists of a high-performance computer, a virtual reality helmet, controllers and a motion capture sensor system. The most suitable is the VR helmet HTC Vive, because it has undeniable advantages over other models presented in the market and the capabilities that are required for the implementation of the project:

- $\quad$ Support for "tracking room" Lighthouse, which allows you to navigate while in virtual reality in a zone up to $4 \times 5$ meters (the other virtual reality glasses are designed for sitting). This allows you to create a greater immersion, thereby maximally approaching the sensation of being in a real laboratory;

- Using the Lighthouse system allows you to effectively track the head in six degrees of freedom. It minimizes the effect of "motion sickness" with a long time in the helmet, which is important;

- $\quad$ Presence in the complete set of controllers.

- $\quad$ Convenience in use with glasses for vision correction, because students wear them;

The general view of the laboratory is equipped with a helmet of virtual reality is presented in Figure 7 and Figure 8, where:

1. The HTC Vive virtual reality helmet, provides a highly detailed stereoscopic image for each eye;

2. The system of motion detectors Lighthouse. It projects the real movements in the movements of the managed virtual character;

3. High-performance laptop is the computational core;

4. Limiter of an accessible area for movement, designed to ensure the safety of others and the person using the helmet.

5. Controllers that allow you to interact with objects in virtual reality (buttons, toggle switches, knobs, curtains, touch panels, etc.)

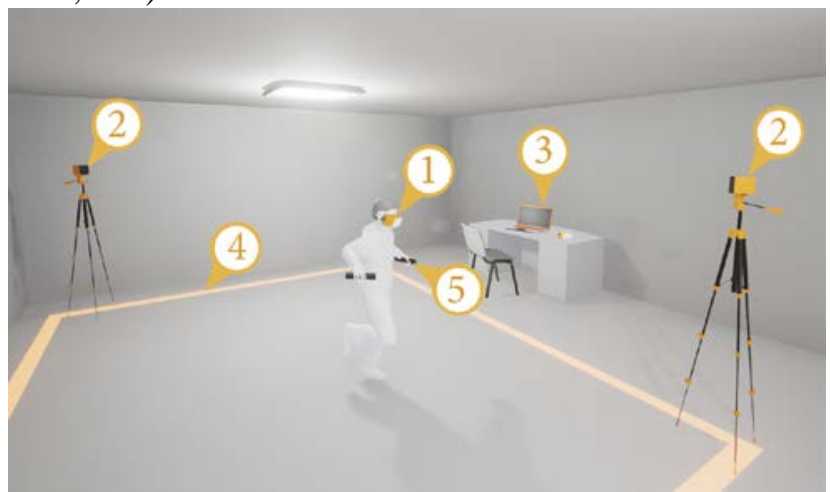

Figure 7. General view of the laboratory which is equipped with a virtual reality helmet.

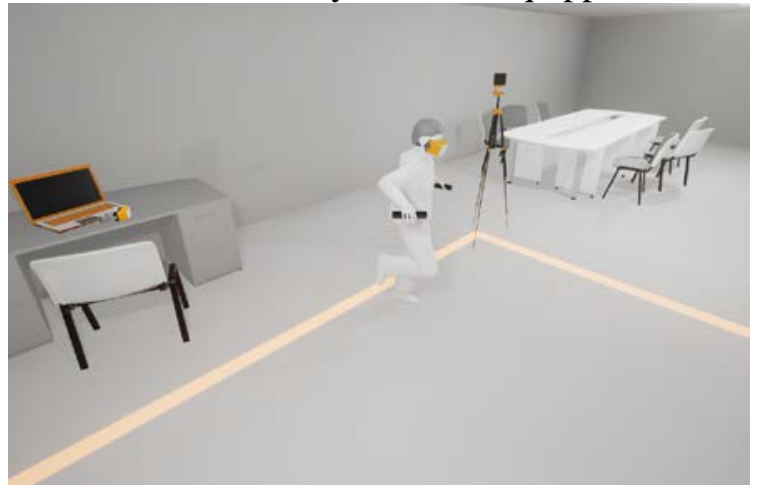

Figure 8. View of the laboratory from the second angle. 
The program part is the cycle of laboratory works, supported by theoretical material, consists of 4-6 laboratory works on various topics of the discipline. The laboratory works are carried out for a small group of students from 6 to 8 people, which allows individualizing the training and controlling the assimilation of the material. Before the beginning of the cycle of laboratory work, it is necessary to provide safety training when person is in the helmet, as well as the basics of control and interaction in a special application that is part of the software package.

Each laboratory work is carried out in stages in the following order:

(If one of the labs has already been completed, then before proceeding to the next, you need to protect the previous one by answering a few test questions)

1. Equipping by a helmet of virtual reality, controllers and checking their functionality;

2. Studying of the theoretical foundations of laboratory work, which are presented in the form of an interactive presentation with sound;

3. Acquaintance with the basics of virtual equipment operation and the order of performing laboratory work;

4. Performing laboratory work;

5. Printing out the results (tables and graphs) obtained during the laboratory work and (or) sending them to the student's e-mail; All results obtained are stored in the database, which prevents the copying of the results of work;

6. Passage of the control test, which confirms the assimilation of the material studied in the course of laboratory work;

7. Drawing up a report on the work done.

During the performance of the laboratory work, the teacher has the opportunity to observe the video stream which are reproduced in the helmet of virtual reality with the aim of correcting the course of laboratory work.

\section{Conclusions}

The use of virtual reality opens a lot of new opportunities in learning and education that are too complex, time-consuming or expensive with traditional approaches, if not all at the same time. There are five main advantages of using VR technologies in education.

- Visibility. Using 3D-graphics, you can detail the chemical processes up to the atomic level. And nothing forbids to go deeper still further and to show how inside the atom the nuclear division is divided before the nuclear explosion. Virtual reality is capable of not only give information about the phenomenon itself, but also demonstrate it with any degree of detail.

- Safety. The work of potentially dangerous equipment and materials - you can immerse the viewer in any of these circumstances without the slightest threat to life

- Involve. Virtual reality allows you to change scenarios, influence to the course of an experiment or solve a mathematical problem in a playable and understandable form.

- Focusing. The virtual world, which will surround the viewer from all sides to all 360 degrees, will allow to concentrate entirely on the material and not be distracted by external stimuli.

- Virtual lessons. The view from the first person and sensation of the adventure In the painted world is one of the main features of virtual reality. It will allow you to conduct lessons entirely in virtual reality.

\section{References}

[1] Annetta L A 2008 Video Games to Education: Why They Should Be Used and How They Are Being Used Theory Into Practice 47(3) 229-239

[2] Rozhkov O V, Piskunov D E, Nosov P A, Pavlov V Yu, Khorokhorov A M and Shirankov A F 2018 Bauman MSTU scientific school "Zoom lens design": features of theory and practice Computer Optics 42(1) 72-83 DOI: 10.18287/2412-6179-2018-42-1-72-83

[3] Kolomiets E I 2017 International Master's degree program "High-Performance and distributed information processing systems" Procedia Engineering 201 832-848 
[4] Kolomiets E I 2017 Analisys of the international Master's degree program "Mathematical Modeling and Information Technologies in Photonics" Procedia Engineering 201 849-862

[5] Kazanskiy N L 2017 Efficiency of deep integration between a research university and an academic institute Procedia Engineering 201 817-831

[6] UnrealEngine 4 Documentation (Access mode: https://docs.unrealengine.com) (28.05.2017)

[7] Sheverev D A and Sanoyan A G 2016 Laboratory stand for the research of hysteresis of linear deformations of piezoelectric elements for the purpose of precision mixing of objects in manometer range Actual Problems of Radio Electronics and Telecommunication 139-141 